\title{
Spatial Intensity Map of HDEMG Based Classification of Muscle Fatigue
}

\author{
Navaneethakrishna MAKARAM ${ }^{\mathrm{a}, 1}$, Sridhar P. ARJUNAN ${ }^{\mathrm{b}}$, Dinesh KUMAR ${ }^{\mathrm{c}}$ and \\ Ramakrishnan SWAMINATHAN ${ }^{\mathrm{a}}$ \\ a Applied Mechanics, Indian Institute of Technology Madras, India \\ ${ }^{\mathrm{b}}$ Electronics and Instrumentation, SRM Institute of Science and Technology, India \\ ${ }^{\mathrm{c}}$ School of Engineering, RMIT University, Melbourne, Australia
}

\begin{abstract}
In this, study, we have investigated to identify the muscle fatigue using spatial maps of High-Density Electromyography (HDEMG). The experiment involves subjects performing plantar flexion at $40 \%$ maximum voluntary contraction until fatigue. During the experiment, HDEMG signal was recorded from the tibialis anterior muscle. The monopolar and bipolar spatial intensity maps were extracted from the HDEMG signal. The random forest classifier with different tree configurations was tested to distinguish nonfatigue and fatigue condition. The results indicate that selected electrodes from the differential intensity map results in an accuracy of $83.3 \%$ with the number of trees set at 17 . This method of spatial analysis of HDEMG signals may be extended to assess fatigue in real life scenarios.
\end{abstract}

Keywords. Muscle fatigue, High-Density EMG, Spatial Maps, Random Forest

\section{Introduction}

High density EMG (HDEMG) is commonly used to investigate the changes in both surface and deep muscle fiber electrical activity. These provide the distribution of motor unit action potential across the muscle fiber. Muscle fatigue, a neuromuscular condition, presents with changes in motor unit action potential duration, conduction velocity and neural active. In this study, the spatial information of HDEMG signals is used to differentiate nonfatigue (NF) and fatigue (F) condition.

\section{Methods}

The study analyzed HDEMG data from nine subjects performing isometric plantar flexion exercise at $40 \%$ maximum voluntary contraction until they are fatigued. HD EMG signals were recorded from the Tibialis anterior muscle using a 16x4 grid of electrodes at a sampling rate of $2441 \mathrm{~Hz}$ [1]. After filtering with a bandpass filter (10$400 \mathrm{~Hz})$ and notch filter $(50 \mathrm{~Hz})$, twenty $(90 \%$ overlapping) windows of 2000 samples length $(820 \mathrm{~ms})$ from the start of the experiment and prior to the end of the experiment is considered as NF $(n=180)$ and $F(n=180)$ segments respectively [1].

\footnotetext{
${ }^{1}$ Navaneethakrishna Makaram, Applied Mechanics - Biomedical Engineering Group, Indian Institute of Technology Madras, Chennai- 600036, India; E-mail: kmakaram@yahoo.com.
} 
Monopolar and Bipolar nonlinear intensity maps (MIM and BIM) are extracted as show in eq 1 and 2. Here, RMS is the root mean squared value, $i$ and $j$ represent the column and row of electrode.

$$
\begin{aligned}
& M I M=\log _{10}\left(\operatorname{RMS}\left(E M G_{i, j}\right)\right) \\
& B I M=\log _{10}\left(R M S\left(E M G_{i, j}-E M G_{i+1, j}\right)\right)
\end{aligned}
$$

The center of gravity (CG), which represents the region of maximum muscle activity, was extracted based on [2]. Columns, which include the CG and two columns of electrode across the CG, are considered as region of interest, and is also utilized for classification. A random forest classifier with varied number of trees $(n=3: 50)$ and a 10fold cross validation scheme is utilized to distinguish NF and F conditions.

\section{Results and Discussion}
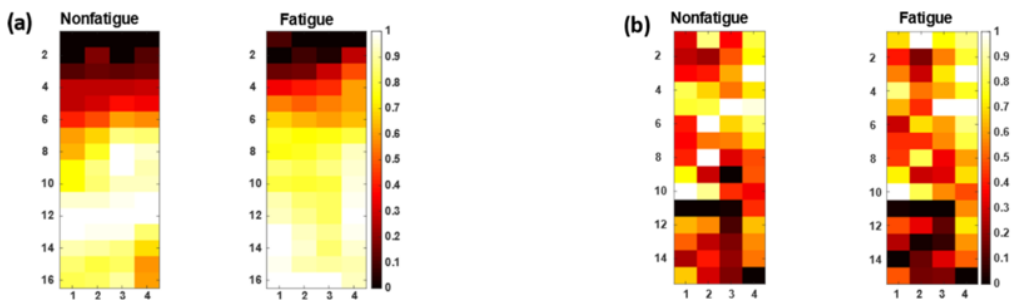

Figure 1. Representative Intensity Maps of HD EMG (a) monopolar and (b) bipolar configuration

Figure 1 (a), represents the monopolar HDEMG maps in NF and F condition is shown. It is observed that a greater number of electrodes present with a high intensity values in F. Figure 1 (b), shows the bipolar HDEMG maps in NF and F condition. It is observed that in the upper electrodes the maps have higher intensity regions. The intensity maps indicate a more uniform potential in $\mathrm{F}$ that might be due to the synchronization of motor unit and the accumulation of lactic acid [3]. The results of the classification indicate that the BIM result in an accuracy of $80.23 \%$ compared to an accuracy of $60 \%$ obtained with MIM. The choice of electrodes closer to CG in BIM result in an improvement in accuracy of $3 \%$.

Table 1. Classification performance of Random Forest for various feature sets

\begin{tabular}{lcccc}
\hline \multicolumn{1}{c}{ Features } & No. of Trees & Accuracy & Sensitivity & Specificity \\
\hline Monopolar Intensity Map (MIM) & 3 & 60.00 & 60.34 & 59.68 \\
Columns of CG (MIM) & 10 & 60.00 & 60.23 & 59.78 \\
Bipolar Intensity Map (BIM) & 14 & 80.28 & 79.78 & 80.79 \\
Columns of CG (BIM) & 17 & 83.33 & 83.71 & 82.97 \\
\hline
\end{tabular}

\section{References}

[1] Bingham A, Arjunan SP, Jelfs B, Kumar DK. Normalised mutual information of high-density surface electromyography during muscle fatigue. Entropy. 2017 Dec;19(12):697.

[2] Jordanić M, Rojas-Martinez M, Mananas MA, Alonso JF. Prediction of isometric motor tasks and effort levels based on high-density EMG in patients with incomplete spinal cord injury. Journal of neural engineering. 2016 May 17;13(4):046002.

[3] Merletti R and Parker P.J, Electromyography: physiology, engineering and noninvasive applications, Wiley-Interscience, Hoboken, NJ; 2010. 